\title{
Practice-based Research Networks (PBRNs) Are Promising Laboratories for Conducting Dissemination and Implementation Research
}

\author{
John Heintzman, MD, MPH, Rachel Gold, PhD, MPH, Alexander Krist, MD, MPH, \\ Jay Crosson, PhD, Sonja Likumahuwa, MID, MPH, Jennifer E. DeVoe, MD, DPhil
}

Dissemination and implementation science addresses the application of research findings in varied health care settings. Despite the potential benefit of dissemination and implementation work to primary care, ideal laboratories for this science have been elusive. Practice-based research networks (PBRNs) have a long history of conducting research in community clinical settings, demonstrating an approach that could be used to execute multiple research projects over time in broad and varied settings. PBRNs also are uniquely structured and increasingly involved in pragmatic trials, a research design central to dissemination and implementation science. We argue that PBRNs and dissemination and implementation scientists are ideally suited to work together and that the collaboration of these 2 groups will yield great value for the future of primary care and the delivery of evidence-based health care. ( $\mathrm{J}$ Am Board Fam Med 2014;27:759-762.)

Keywords: Dissemination and Implementation Science, Evidence-Based Medicine, Practice-based Research, Pragmatic Trials, Primary Health Care

Dissemination and implementation (D\&I) science addresses the multilevel elements of health care delivery that affect the translation of effective interventions, strategies, or practices between care settings. ${ }^{1,2}$ Dissemination can generally be defined as the "targeted distribution of information and inter-

This article was externally peer reviewed.

Submitted 13 March 2014; revised 16 June 2014; accepted 23 June 2014.

From the Department of Family Medicine, Oregon Health and Science University, Portland (JH, SL, JED); the Kaiser Center for Health Research (RG); Virginia Commonwealth University (AK); and Mathematica Policy Research (JC).

Funding: Support for this manuscript came from the Agency for Healthcare Quality and Research (grant no. K08HS021522); the Health Resources and Services Administration (HRSA) (grant no. UB2HA20235); the National Institutes of Health (NIH) National Library of Medicine (grant no. RC4LM010852); and the NIH National Heart, Lung and Blood Institute (grant no. R18HL095481). Additional support for the project was provided by the Department of Family Medicine, Oregon Health \& Science University, Portland.

Conflict of interest: JED is an editorial board member of the $7 A B F M$.

Corresponding author: John Heintzman, MD, MPH, Department of Family Medicine, Oregon Health and Science University, 3181 SW Sam Jackson Park Rd, Portland, OR 97239 (E-mail: heintzma@ohsu.edu). vention materials to a specific public health or clinical practice audience" and implementation as "the use of strategies to adopt and integrate evidencebased health interventions and change practice patterns within specific settings." ${ }^{3}$ While the need to improve evidence-based health care is well acknowledged, D\&I researchers must address considerable challenges, such as negotiating complex, real-world practice settings in which to conduct their work. We argue that D\&I scientists have not yet fully utilized a promising resource for meeting these challenges: the practice-based research network (PBRN). Because PBRNs and D\&I scientists share similar aims, including a desire to understand what works in "real-world" settings, the natural partnership between them should be further developed and evaluated.

\section{D\&I Research Needs "Real-World" Laboratories}

In contrast to translational research, which aims to "translate" basic science findings to clinical ones in efficacy trials, D\&I scientists ask how best to apply findings obtained in controlled research environ- 
ments to real-world settings. D\&I investigators have encountered difficulties in finding laboratories in which to conduct this type of research ${ }^{4-9}$-laboratories that enable them to balance effectively the need for internal validity (the standardization of research protocol and minimization of bias) and external validity (the applicability of research interventions and outcomes to the real health of patients). Academic medical centers have research experience and infrastructure but are not representative of real-world practices and populations; thus, D\&I scientists must look beyond these traditional research settings. ${ }^{10}$ Community settings are more "real world" but focus, by design, on providing clinical care, not conducting research. ${ }^{1-13}$ While clinic staff in community settings certainly have interest in research applicable to their patients and practices, their clinical tasks take priority over participating in and adhering to research protocols. $^{12}$

D\&I researchers need "community laboratories" with a well-organized infrastructure of clinical partners who are accustomed to participating in research and knowledgeable about applying findings into practice. Ideally, these partners should be from diverse settings and regions to maximize external validity. PBRNs are just such a setting. While there are several examples of successful D\&I research in PBRN-like settings that have produced meaningful results, ${ }^{14,15}$ PBRNs could be used on a much more significant scale to conduct D\&I research.

\section{PBRNs: A Promising Setting for D\&I}

As defined by the Agency for Health care Quality and Research, PBRNs are "groups of primary care clinicians and practices working together to answer community-based health care questions and translate research findings into practice." 16 PBRNs are particularly well positioned to support and execute studies that ask whether interventions that are effective in one setting can be implemented in a different setting and how best to accelerate the diffusion of evidence-based innovations into everyday practice-the questions most central to the field of D\&I. ${ }^{17}$ Unlike other research settings, PBRNs involve relationships between researchers and community practitioners from networks of real-world practices-relationships that address the challenges of D\&I science.
The organizational structures of mature and productive PBRNs offer several advantages for D\&I research. First, longitudinal relationships between practices executing multiple projects enable the improvement of research processes over time, so that clinician-researcher partnerships need not reinvent the wheel, so to speak, with every new study. This can build trust, increase efficiency, streamline communication, and improve the ability to adapt methods/protocols to different settings. Practices and clinicians play a more active role in producing high-quality research applicable to their patients, which may also streamline patient recruitment into new initiatives. Researcher-community clinician partnerships also are crucial to informing all stages of research. Real-world observations are necessary to properly frame research questions, to focus questions and methods on hypotheses with clinical significance and patient-centered impacts, and to interpret findings for the community setting. This is increasingly recognized by funders; current funding opportunities involving D\&I from the Agency for Health care Quality and Research call for the participation of PBRN-type networks. ${ }^{18}$ Collaboration over time also specifically facilitates pragmatic trials, a study design crucial to D\&I research.

\section{Pragmatic Trials: A Strategic PBRN Strength}

A particular strength of PBRNs is their ability to participate in pragmatic trials, which differ from standard clinical trials in that they are performed in real-world clinical environments and account for variation in routine clinical practice. Pragmatic trials are essential to testing the translation of experimental findings into heterogeneous settings and to balancing internal and external validity. ${ }^{19-21}$ PBRNs have a long history not only of successful completion of effective observational studies ${ }^{22}$ but also of increasingly pragmatic trials in such content areas as preventive care, asthma management, and osteoporosis screening. ${ }^{23-27}$ This growing record further supports the strategic position of PBRNs in advancing D\&I science. Despite this promise, the medical literature contains minimal mention of partnerships between these 2 disciplines. The execution of multiple D\&I studies in PBRNs is necessary to move both fields forward and demonstrate a sustainable partnership. 


\section{The Benefits of DEI for PBRNs}

As noted earlier, many academic investigators conduct research in controlled experimental settings that are minimally relevant to community practices, and PBRNs have long been addressing how to apply medical evidence to community practice. D\&I research involves theoretical frameworks and approaches developed specifically for community settings. ${ }^{28-30}$ PBRNs may benefit greatly from partnering with scientists with expertise in this pragmatic discipline, allowing them to continue to develop novel ways to approach everyday clinical problems, answer difficult practice questions, and improve the delivery of care overall. D\&I scientists and others wishing to conduct pragmatic trials with a high degree of external validity will benefit greatly from partnering with PBRNs to help inform policies and procedures for pragmatic trials, to achieve minimal disruption of clinical practice, and to design and execute studies in ways that will ensure sustainability after the trial ends.

\section{Conclusion}

Enhancing the evidence base underlying our health care system requires better information on how to disseminate and implement experimental findings. To date, D\&I science has struggled to find successful settings in which to address this need. PBRNs provide a promising setting for D\&I studies because they bring together the appropriate relational networks and experience participating in research. We strongly suggest that D\&I scientists and practice-based researchers join forces to accelerate the application of evidence into practice in diverse realworld settings.

\section{References}

1. Glasgow RE, Vinson C, Chambers D, Khoury MJ, Kaplan RM, Hunter C. National Institutes of Health approaches to dissemination and implementation science: current and future directions. Am J Public Health 2012;102:1274-81.

2. Collins FS. Research agenda. Opportunities for research and NIH. Science 2010;327:36-7.

3. Dissemination and implementation research in health (R01). Bethesda (MD): National Institutes of Health; 2012. Available from: http://grants.nih.gov/grants/ guide/pa-files/PAR-10-038.html. Accessed September 16, 2014.

4. Teal R, Bergmire DM, Johnston M, Weiner BJ. Implementing community-based provider participa- tion in research: an empirical study. Implement Sci 2012;7:41.

5. van Tulder MW, Croft PR, van Splunteren P, et al. Disseminating and implementing the results of back pain research in primary care. Spine (Phila $\mathrm{Pa} 1976$ ) 2002;27:E121-7.

6. Grimshaw JM, Shirran L, Thomas R, et al. Changing provider behavior: an overview of systematic reviews of interventions. Med Care 2001;39(8 Suppl 2):II2-45.

7. Damschroder LJ, Aron DC, Keith RE, Kirsh SR, Alexander JA, Lowery JC. Fostering implementation of health services research findings into practice: a consolidated framework for advancing implementation science. Implement Sci 2009;4:50.

8. Titler MG, Wilson DS, Resnick B, Shever LL. Dissemination and implementation: INQRI's potential impact. Med Care 2013;51(4 Suppl 2):S41-6.

9. Bodenheimer T, Young DM, MacGregor K, Holtrop JS. Practice-based research in primary care: facilitator of, or barrier to, practice improvement? Ann Fam Med 2005;3(Suppl 2):S28-32.

10. Green LA, Fryer GE Jr, Yawn BP, Lanier D, Dovey SM. The ecology of medical care revisited. N Engl J Med 2001;344:2021-5.

11. Calmbach WL, Ryan JG, Baldwin LM, Knox L. Practice-based research networks (PBRNs): meeting the challenges of the future. J Am Board Fam Med 2012;25:572-6.

12. Heintzman J, Likumahuwa S, Nelson C, et al. "Not a kidney or a lung": research challenges in a network of safety net clinics. Fam Med Feb 2014;46:105-11.

13. Bertram S, Graham D, Kurland M, Pace W, Madison S, Yawn BP. Communication is the key to success in pragmatic clinical trials in practice-based research networks (PBRNs). J Am Board Fam Med 2013;26:571-8.

14. Rebchook GM, Kegeles SM, Huebner D, Team TR. Translating research into practice: the dissemination and initial implementation of an evidence-based HIV prevention program. AIDS Educ Prev 2006; 18(4 Suppl A):119-36.

15. Dudl RJ, Wang MC, Wong M, Bellows J. Preventing myocardial infarction and stroke with a simplified bundle of cardioprotective medications. Am J Manag Care. 2009; 15:e88-94.

16. Practice-based research networks (PBRNs). Rockville (MD): Agency for Healthcare Quality and Research; 2014. Available from: http://pbrn.ahrq.gov/. Accessed March 10, 2014.

17. Peterson KA, Lipman PD, Lange CJ, Cohen RA, Durako S. Supporting better science in primary care: a description of practice-based research networks (PBRNs) in 2011. J Am Board Fam Med 2012;25: 565-71.

18. Accelerating the dissemination and implementation of PCOR findings into primary care practice (R18). Rockville (MD): Agency for Healthcare Research 
and Quality; 2014. Available from: http://grants.nih. gov/grants/guide/rfa-files/RFA-HS-14-008.html. Accessed September 16, 2014.

19. Fransen GAJ, van Marrewijk CJ, Mujakovic S, et al. Pragmatic trials in primary care. Methodological challenges and solutions demonstrated by the DIAMOND-study. BMC Med Res Methodol 2007;7:16.

20. Godwin M, Ruhland L, Casson I, et al. Pragmatic controlled clinical trials in primary care: the struggle between external and internal validity. BMC Med Res Methodol 2003;3:28.

21. Using pragmatic clinical trials to test the effectiveness of patient-centered medical home models in real-world settings. Rockville (MD): Agency for Healthcare Quality and Research; 2013.

22. Green LA, Hames CG Sr, Nutting PA. Potential of practice-based research networks: experiences from ASPN. Ambulatory Sentinel Practice Network. J Fam Pract 1994;38:400-6.

23. Coronado GD, Vollmer WM, Petrik A, et al. Strategies and opportunities to STOP colon cancer in priority populations: pragmatic pilot study design and outcomes. BMC Cancer 2014;14:55.

24. Levy BT, Hartz A, Woodworth G, Xu Y, Sinift S. Interventions to improving osteoporosis screening: an Iowa Research Network (IRENE) study. J Am Board Fam Med 2009;22:360-7.
25. Krist AH, Glenn BA, Glasgow RE, et al. Designing a valid randomized pragmatic primary care implementation trial: the my own health report (MOHR) project. Implement Sci 2013;8:73.

26. Daniels EC, Bacon J, Denisio S, et al. Translation squared: improving asthma care for high-disparity populations through a safety net practice-based research network. J Asthma 2005;42:499-505.

27. Coronado GD, Sanchez J, Petrik A, Kapka T, Devoe J, Green B. Advantages of wordless instructions on how to complete a fecal immunochemical test: lessons from patient advisory council members of a federally qualified health center. J Cancer Educ 2014;29:86-90.

28. Tabak RG, Khoong EC, Chambers DA, Brownson RC. Bridging research and practice: models for dissemination and implementation research. Am J Prev Med 2012;43(3):337-50.

29. Re-Aim. Resources and tools. Blacksburg (VA): Department of Human Nutrition, Foods, and Exercise, Virginia Tech; 2014. Available from: http://www.reaim.org/resources_and_tools/index.html. Accessed September 16, 2014.

30. The North Carolina Translational and Clinical Sciences Institute. Dissemination and implementation theories and frameworks. Available from: http://tracs.unc.edu/index. $\mathrm{php} / \mathrm{d}$-iportal/theories-and-frameworks. Accessed June 14, 2014. 